\title{
Enantioselective total synthesis of naturally occurring eushearilide and evaluation of its antifungal activity
}

\author{
Takayuki Tonoi, Ryo Kawahara, Takehiko Inohana and Isamu Shiina
}

The asymmetric total synthesis of a newly proposed structure of $(3 S, 16 E, 20 E, 23 S)-(+)$-eushearilide was achieved primarily through an asymmetric Mukaiyama aldol reaction, Schlosser-modified Wittig reaction and 2-methyl-6-nitrobenzoic anhydridemediated macrolactonization. Based on detailed spectroscopic analyses, the obtained synthetic compound was found to be identical to natural eushearilide. Therefore, we were able to determine the true structure of eushearilide. Moreover, the synthetic compound was found to exhibit significant in vitro antifungal activity against various fungi and bacteria.

The Journal of Antibiotics (2016) 69, 697-701; doi:10.1038/ja.2015.146; published online 27 January 2016

\section{INTRODUCTION}

Eushearilide was first isolated from a culture of the fungus Eupenicillium shearii and structurally characterized in 2006 by Hosoe et al. ${ }^{1}$ (Figure 1, structure 1). This was found to be a very useful natural product as it exhibits a broad range of antifungal activity against diverse fungi and yeasts, such as the human pathogens Aspergillus fumigatus, Trichophyton spp. and Candida spp. The originally proposed planar structure of eushearilide (1) was shown to consist of a 24-membered lactone framework as the main part bearing two stereogenic centers (C3 and C23), whose absolute configurations were not determined. Moreover, the present compound was proposed to have a nonconjugated diene system ( $16 Z$ and $20 E$ ) and a phosphorylcholine group. This is in contrast to typical polyene macrolides, which generally contain a conjugated polyene structure and a 1,3polyol section, such as in the antifungal drugs amphotericin B, nystatin and pimaricin. These unique structural characteristics and a promising structure-activity relationship (SAR) prompted us to stereoselectively synthesize $\mathbf{1}$ in order to determine the absolute stereostructure of naturally occurring eushearilide and accelerate its SAR studies. While researching the synthesis of eushearilide, ${ }^{2-5}$ Higashiyama et al. ${ }^{6}$ reported the first total synthesis of 1 , but they stated that the synthetic product was not identical to the natural compound. However, we achieved the asymmetric total synthesis of $(3 R, 23 R)-\mathbf{1}$ and its diastereomer $((3 S, 23 R)-\mathbf{1})$ mainly through an asymmetric aldol reaction and 2-methyl-6-nitrobenzoic anhydride $(\mathrm{MNBA})^{7-14}$ mediated macrolactonization in the presence of a nucleophilic catalyst such as 4-(dimethylamino)pyridine (DMAP) or 4-(dimethylamino)pyridine $\mathrm{N}$-oxide. ${ }^{15}$ Based on detailed NMR analyses of these synthetic compounds, we revealed that natural eushearilide does not possess a $(16 Z, 20 E)$ configuration but rather a $(16 E, 20 E)$ configuration. We also successfully synthesized $(3 R, 16 E, 20 E, 23 R)$ (-)-eushearilide, whose optical rotation sense was found to be opposite to that of the natural product. ${ }^{15}$ We report herein the enantioselective total synthesis of a newly proposed structure of $(3 S, 16 E, 20 E, 23 S)$-eushearilide (2) and evaluation of its antimicrobial activity.

\section{RESULTS AND DISCUSSION}

In order to synthesize 2, we first performed the transformation to desired seco acid 13 starting from aldehyde 3 with phosphonium salt 4 (Scheme 1; For experimental procedures of $\mathbf{3}$ and $\mathbf{4}$, see Supplementary Information). Stereoselective formation of the $(E)$ olefin moiety at C16-C17 in 2 could be achieved via a trans-selective modified Wittig olefination, also known as the Schlosser modification. ${ }^{16-18}$ Transformation of aldehyde 3 with phosphonium salt 4 via the modified Wittig reaction was performed to afford desired coupling product $\mathbf{5}$ in good yield exclusively in the trans-form. The configuration of the double bond (C8-C9) in $\mathbf{5}$ was assigned as $E$ based on the downfield shifts of the allylic methylene $\left(\delta_{\mathrm{C}} 32.6(\mathrm{C} 7)\right.$ and $\left.\delta_{\mathrm{C}} 32.6(\mathrm{C} 10)\right)$, while the $Z$-configuration was deduced from the upfield chemical shifts of the allylic methylene $\left(\delta_{\mathrm{C}} 27.3(\mathrm{C} 7)\right.$ and $\delta_{\mathrm{C}}$ $27.2(\mathrm{C} 10)) .{ }^{19-22}$ Successive deprotection of the terminal TBS group in 5 and subsequent oxidation of obtained alcohol 6 afforded corresponding aldehyde $\mathbf{7}$ in high yield. Aldol product $\mathbf{9}$, which possesses a $(3 S, 23 S)$ configuration, was stereoselectively synthesized via the asymmetric Mukaiyama aldol reaction ${ }^{23-25}$ of 1-ethylthio-1-(trimethylsiloxy)ethene (8), which was derived from $S$-ethyl propanethioate, with obtained aldehyde 7 in the presence of an (R)-diamine-Sn(II) complex as the catalyst with ${ }^{n} \mathrm{Bu}_{3} \mathrm{SnF}$. Next, the transesterification of thioester 9 with silver trifluoroacetate was performed to provide corresponding ethyl ester 10. Consecutive $p$-methoxybenzyl (PMB) protection of the secondary hydroxyl group in $\mathbf{1 0}$ and deprotection of the BOM group followed by saponification of the ethyl ester moiety yielded desired seco acid 13, a precursor of the ring-closing product, in good yield. 


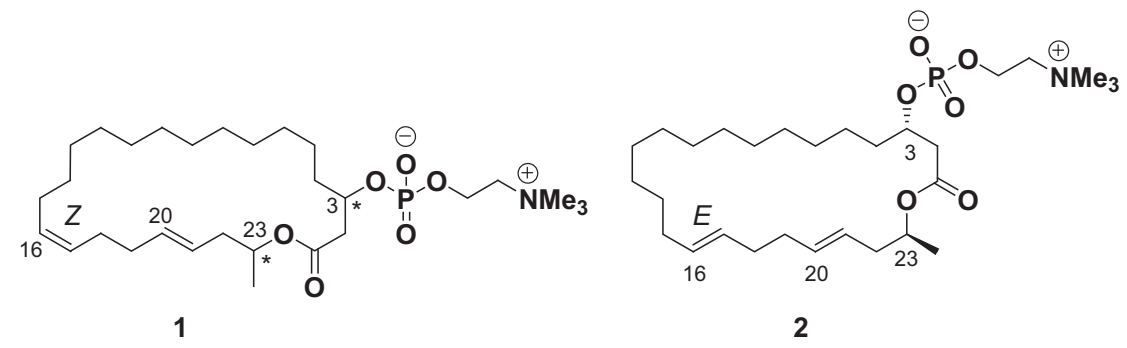

Figure 1 Original (1) and new (2) proposed structures of eushearilide.

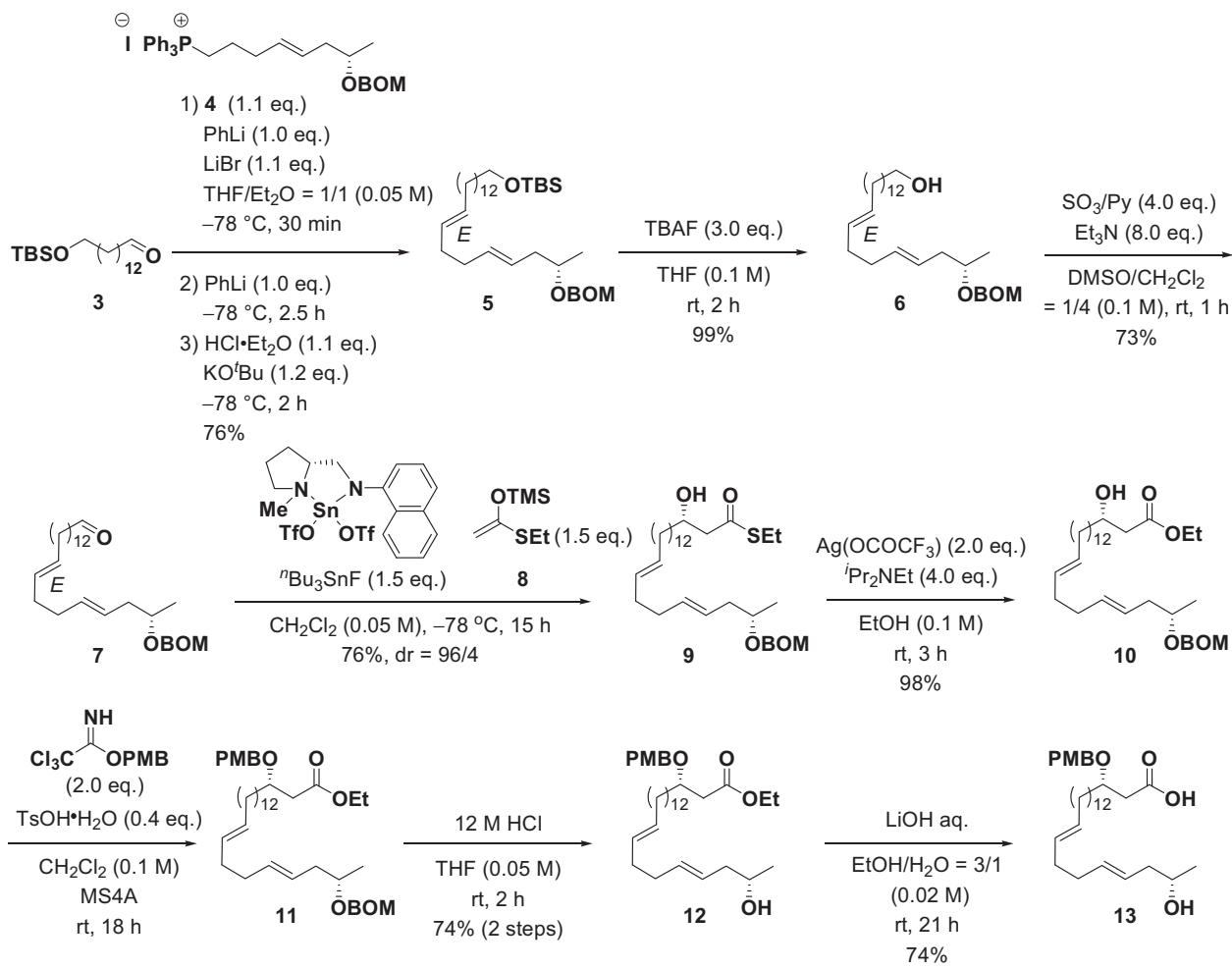

Scheme 1 Transformation of $\mathbf{3}$ to seco acid $\mathbf{1 3}$

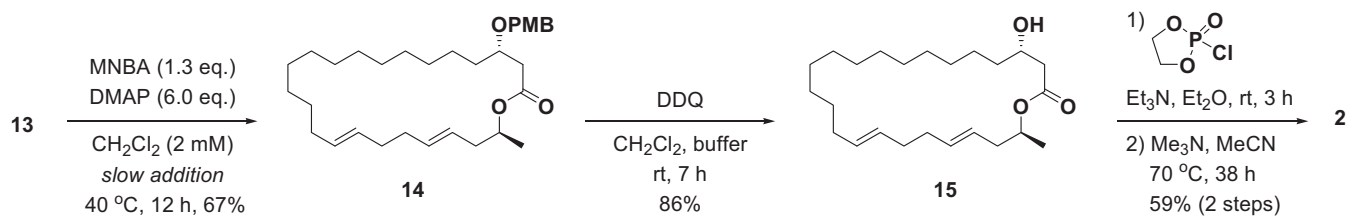

Scheme 2 Transformation of 13 to the naturally occurring eushearilide (2).

Next, we attempted the macrolactonization of seco acid $\mathbf{1 3}$ via the MNBA method in the presence of DMAP as a nucleophilic catalyst (Scheme 2). ${ }^{7-15}$ An excess amount (6.0 eq.) of DMAP was employed with MNBA in dichloromethane at room temperature to afford 24 -membered lactone $\mathbf{1 4}$ in $67 \%$ yield. After the successful macrolactonization using MNBA, a choline residue was attached to the obtained macrolactone framework. Following deprotection of the $\mathrm{PMB}$ group in 14 with 2,3-dichloro-5,6-dicyano-1,4-benzoquinone, a phosphorylcholine moiety ${ }^{26}$ was introduced on the secondary hydroxyl group in $\mathbf{1 5}$ to yield the desired $(3 S, 16 E, 20 E, 23 S)-2$.
The ${ }^{1} \mathrm{H}$ and ${ }^{13} \mathrm{C}$ NMR spectra of the desired compound 2 were then compared with those of the natural product (Table 1). Owing to the newly stereoselective construction of the (E)-olefin moiety at C16-C17 in $\mathbf{2}$, the chemical shifts of all carbons in $\mathbf{2}$ were in complete agreement with those of the natural product reported in the literature, ${ }^{1}$ as clearly shown in Figure 2. Moreover, the optical rotation sense of $2\left([\alpha]_{\mathrm{D}}{ }^{25}+11.3(c 0.85, \mathrm{MeOH})\right)$ was consistent with that reported for naturally occurring eushearilide $\left([\alpha]_{\mathrm{D}}{ }^{25}+12.8\right.$ (c 0.75 , $\mathrm{MeOH}))^{1}$ From these results, we were able to determine the complete stereostructure of eushearilide. 
Table $1{ }^{1} \mathrm{H}$ and ${ }^{13} \mathrm{C}$ NMR shifts (p.p.m.) of natural and synthetic eushearilide (2) in $\mathrm{CDCl}_{3}$

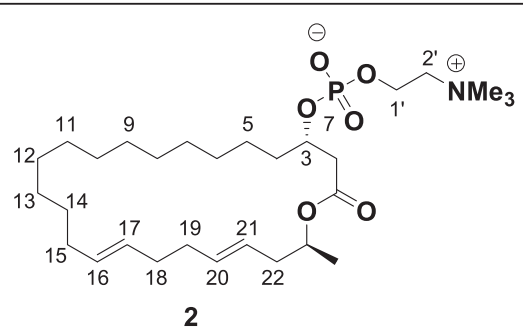

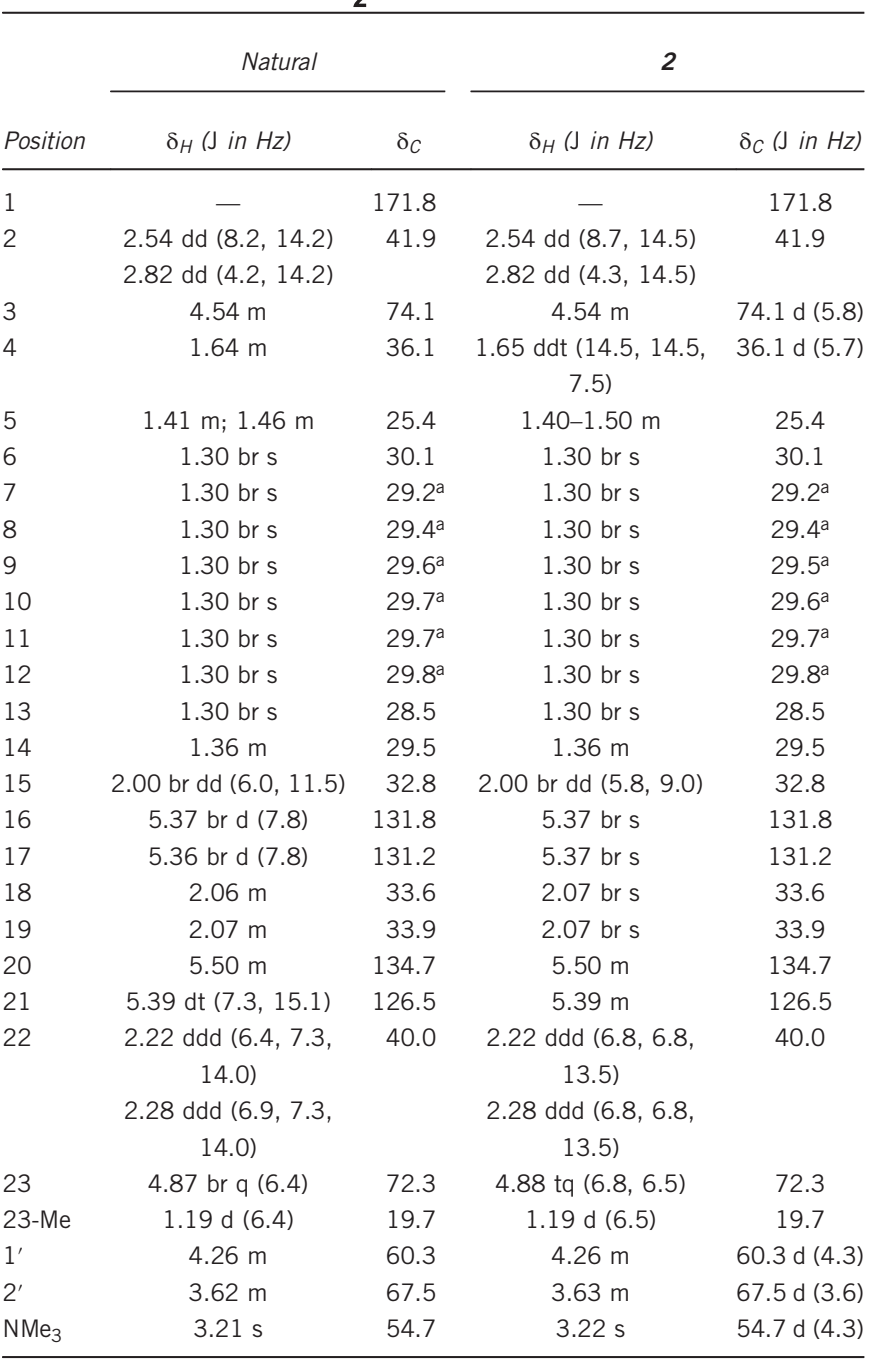

aCarbon chemical shifts might be interchanged.

Finally, the in vitro antimicrobial activities of synthetic compound 2 and $(3 R, 23 R)-\mathbf{1}$, a stereoisomer of the originally proposed structure $\mathbf{1}$, against various fungi and bacteria were assayed using the disk diffusion method according to the Clinical and Laboratory Standards Institute. ${ }^{27,28}$ The inhibition zones were measured in millimeters against each microorganism, as shown in Table 2. The inhibition diameters of 2 against pathogenic bacteria Staphylococcus aureus (NBRC 12732) and pathogenic filamentous fungi Aspergillus niger (NBRC 105649) were 1.3 and $4.3 \mathrm{~mm}$, respectively, at a concentration of $50 \mu \mathrm{g}$ per disk. Moreover, the antimicrobial activity level of 2 against pathogenic filamentous fungi Trichophyton mentagrophytes (NBRC 5466) was relatively high (17.3 mm diameter) compared with the above two microorganisms. These results are well in line with the

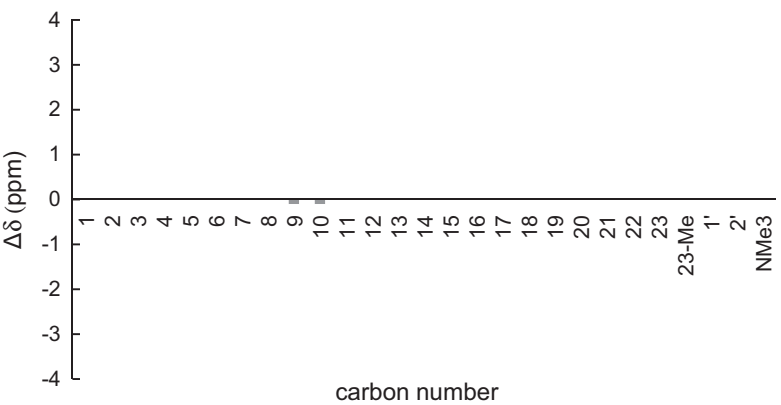

Figure $2 \Delta \delta$ (p.p.m.) of the ${ }^{13} \mathrm{C} N M R$ chemical shifts of $(3 S, 16 E, 20 E, 23 S$ )2 in $\mathrm{CDCl}_{3} . \Delta \delta$ corresponds to the difference in the chemical shift of the natural and synthetic products $(\Delta \delta=\delta$ (synthetic) $-\delta$ (natural)).

Table 2 Antimicrobial activities of synthetic eushearilide (2) and original structure of eushearilide $(3 R, 23 R)-1$

\begin{tabular}{|c|c|c|c|}
\hline \multirow[b]{2}{*}{ Microorganisms } & & \multicolumn{2}{|c|}{ Inhibition zone $\mathrm{e}^{\mathrm{a}}(\mathrm{mm})$} \\
\hline & & 2 & $(3 R, 23 R)-1$ \\
\hline Staphylococcus aureus & NBRC 12732 & 1.3 & 2.2 \\
\hline Aspergillus niger & NBRC 105649 & 4.3 & 3.7 \\
\hline Trichophyton mentagrophytes & NBRC 5466 & 17.3 & 6.3 \\
\hline
\end{tabular}

aThe clear zone of inhibition $(\mathrm{mm})$ around a paper disk impregnated with an antimicrobial agent at a concentration of $50 \mu \mathrm{g}$ per disk.

reported data in the literature ${ }^{1}$ and exhibit the same or higher level of activity compared with the general antimicrobial agents, such as trichlosan, commonly used as a chemical drug for sterilization. ${ }^{29}$ Moreover, $(3 R, 23 R)-\mathbf{1}$ was also found to show the same tendency as in the case of compound 2. Therefore, the geometrical isomeric structure of the olefin moiety at $\mathrm{C} 16-\mathrm{C} 17$ might not affect antimicrobial activity of eushearilide. Thus synthetic compound 2 was found to clearly exhibit antifungal activity against various microorganisms, as described in the literature. ${ }^{1}$

In conclusion, we successfully achieved the first asymmetric total synthesis of naturally occurring eushearilide (2) and elucidated its true structure through detailed spectroscopic analyses; that is, (3S,16E,20E,23S)-(+)-eushearilide. Moreover, we confirmed that $(3 S, 16 E, 20 E, 23 S)-2$ demonstrated the expected antimicrobial activity against various fungi and bacteria.

\section{MATERIALS AND METHODS}

\section{General experimental procedures}

IR spectra were obtained using a Horiba FT-300 Fourier transform IR spectrometer (Horiba Ltd., Kyoto, Japan). NMR spectra were recorded on the following instrument: JEOL JNM-AL500 $\left({ }^{1} \mathrm{H}\right.$ at $500 \mathrm{MHz}$ and ${ }^{13} \mathrm{C}$ at $125 \mathrm{MHz}$; JEOL Ltd., Tokyo, Japan). Optical rotations were determined using a Jasco P-1020 polarimeter (JASCO Ltd., Tokyo, Japan). Mass spectra were determined by a Bruker Daltonics micrOTOF focus (ESI-TOF) mass spectrometer (Bruker Daltonics Inc., Billerica, MA, USA). HPLC was performed with a Hitachi LaChrom Elite system composed of the Organizer (Hitachi HighTechnologies Corporation, Tokyo, Japan), L-2400 UV Detector and L-2130 Pump. All reactions were carried out under argon atmosphere in dried glassware unless otherwise noted.

Experimental procedures and characterization data

Experimental procedures of all new compounds are summarized in Supplementary Information. 
(2S,4E,8E)-2-Benzyloxymethoxy-22-tert-butyldimethylsilyloxy-docosa-4,8-diene (5). ${ }^{1} \mathrm{H}-\mathrm{NMR}\left(500 \mathrm{MHz}, \mathrm{CDCl}_{3}\right): \delta 7.33(\mathrm{~m}, 5 \mathrm{H}, \mathrm{BOM}), 5.51-5.38(\mathrm{~m}, 4 \mathrm{H}$, $4-\mathrm{H}, 5-\mathrm{H}, 8-\mathrm{H}, 9-\mathrm{H}), 4.79$ (dd, $J=6.9,14.6 \mathrm{~Hz}, 2 \mathrm{H}, \mathrm{BOM}), 4.62$ (dd, $J=12.0$, $15.5 \mathrm{~Hz}, 2 \mathrm{H}, \quad$ BOM), 3.79 (ddd, $J=6.3,12.3,12.5 \mathrm{~Hz}, 1 \mathrm{H}, 2-\mathrm{H}$ ), 3.59 $(\mathrm{t}, J=6.3 \mathrm{~Hz}, 2 \mathrm{H}, 22-\mathrm{H}$ ), 2.22 (ddd, $J=6.3,6.9,10.0 \mathrm{~Hz}, 1 \mathrm{H}, 3-\mathrm{H}$ ), 2.17 (ddd, $J=6.3,6.9,10.0 \mathrm{~Hz}, 1 \mathrm{H}, 3-\mathrm{H}), 2.05$ (br s, 4H, 6-H, 7-H), 1.95 (dd, $J=6.9,12.3 \mathrm{~Hz}, 2 \mathrm{H}, 10-\mathrm{H}), 1.51(\mathrm{tt}, J=6.9 \mathrm{~Hz}, 2 \mathrm{H}, 21-\mathrm{H}), 1.31-1.25(\mathrm{~m}, 20 \mathrm{H}$, $11-\mathrm{H}$ to $20-\mathrm{H}$ ), 1.17 (d, $J=6.3 \mathrm{~Hz}, 3 \mathrm{H}, 1-\mathrm{H}$ ), 0.897 (s, 9H, TBS), 0.056 (s, 6H, TBS). ${ }^{13} \mathrm{C}-\mathrm{NMR}\left(125 \mathrm{MHz}, \mathrm{CDCl}_{3}\right): \delta 138.0$ (BOM), 132.7 (C5), 130.8 (C9), 129.5 (C8), $128.4(\mathrm{BOM}), 127.8(\mathrm{BOM}), 127.6(\mathrm{BOM}), 126.2(\mathrm{C} 4), 92.8$ (BOM), 73.0 (C2), 69.3 (BOM), 63.4 (C22), 40.1 (C3), 32.9 (C21), 32.8 (C6), 32.6 (C7), 32.6 (C10), 29.7, 29.6, 29.6, 29.5, 29.2, 25.8 (C11 to C20), 26.0 (TBS), 19.9 (C1), 18.4 (TBS), -5.23 (TBS). HR-MS (ESI-TOF): $\mathrm{m} / z$ calcd for $\mathrm{C}_{36} \mathrm{H}_{64} \mathrm{O}_{3} \mathrm{SiNa}[\mathrm{M}+\mathrm{Na}]^{+}$595.4517, found 595.4494. IR (neat): 2923, $2854 \mathrm{~cm}^{-1} \cdot[\alpha]_{\mathrm{D}}^{23}-3.63\left(c 1.00, \mathrm{CHCl}_{3}\right)$.

(21S, 14E,18E)-21-Benzyloxymethoxydocosa-14,18-dien-1-ol (6). ${ }^{1} \mathrm{H}-\mathrm{NMR}$ $\left(500 \mathrm{MHz}, \mathrm{CDCl}_{3}\right): \delta 7.36-7.27(\mathrm{~m}, 5 \mathrm{H}, \mathrm{BOM}), 5.51-5.34(\mathrm{~m}, 4 \mathrm{H}, 14-\mathrm{H}$, $15-\mathrm{H}, 18-\mathrm{H}, 19-\mathrm{H}), 4.79$ (dd, $J=7.5,14.3 \mathrm{~Hz}, 2 \mathrm{H}, \mathrm{BOM}), 4.61$ (dd, $J=11.5$, $15.5 \mathrm{~Hz}, 2 \mathrm{H}, \mathrm{BOM}), 3.79$ (ddd, $J=6.3,12.3,12.6 \mathrm{~Hz}, 1 \mathrm{H}, 21-\mathrm{H}), 3.64$ (t, $J=6.9 \mathrm{~Hz}, 2 \mathrm{H}, 1-\mathrm{H}$ ), 2.28 (ddd, $J=6.3,6.9,10.0 \mathrm{~Hz}, 1 \mathrm{H}, 20-\mathrm{H}), 2.16$ (ddd, $J=6.3,6.9,10.0 \mathrm{~Hz}, 1 \mathrm{H}, 20-\mathrm{H}$ ), 2.04 (br s, $4 \mathrm{H}, 16-\mathrm{H}, 17-\mathrm{H}$ ), 1.95 (dd, $J=6.9,12.3 \mathrm{~Hz}, 2 \mathrm{H}, 13-\mathrm{H}), 1.56(\mathrm{tt}, J=6.9,6.9 \mathrm{~Hz}, 2 \mathrm{H}, 2-\mathrm{H}), 1.31-$ $1.25(\mathrm{~m}, 20 \mathrm{H}, 3-\mathrm{H}$ to $12-\mathrm{H}), 1.17(\mathrm{~d}, J=6.3 \mathrm{~Hz}, 3 \mathrm{H}, 22-\mathrm{H}) .{ }^{13} \mathrm{C}-\mathrm{NMR}$ (125 MHz, $\left.\mathrm{CDCl}_{3}\right): \delta 138.0$ (BOM), 132.7 (C18), 130.8 (C14), 129.5 (C15), 128.4 (BOM), 127.9 (BOM), 127.6 (BOM), 126.2 (C19), 92.8 (BOM), 73.0 (C21), 69.3 (BOM), 63.1 (C1), 53.9 (C2), 40.1 (C20), 32.8 (C17), 32.6 (C16), 32.6 (C13), 29.6, 29.6, 29.5, 29.4, 29.4, 29.2, 29.1, 25.7, 20.8 (C4 to C12), 14.1 (C3), 19.9 (C22). HR-MS (ESI-TOF): $m / z$ calcd for $\mathrm{C}_{30} \mathrm{H}_{50} \mathrm{O}_{3} \mathrm{Na}$ $[\mathrm{M}+\mathrm{Na}]^{+} 481.3652$, found 481.3659. IR (neat): 3402, 2923, $2854 \mathrm{~cm}^{-1}$. [ $\left.\alpha\right]$ $\mathrm{D}^{24}-3.28\left(\right.$ c $\left.1.01, \mathrm{CHCl}_{3}\right)$.

(21S,14E,18E)-21-Benzyloxymethoxydocosa-14,18-dienal

(7). ${ }^{1} \mathrm{H}-\mathrm{NMR}$ $\left(500 \mathrm{MHz}, \mathrm{CDCl}_{3}\right): \delta 9.65(\mathrm{t}, J=1.7 \mathrm{~Hz}, 1 \mathrm{H}, 1-\mathrm{H}), 7.27-7.18(\mathrm{~m}, 5 \mathrm{H}$, BOM), $5.43-5.30(\mathrm{~m}, 4 \mathrm{H}, 14-\mathrm{H}, 15-\mathrm{H}, 18-\mathrm{H}, 19-\mathrm{H}), 4.70(\mathrm{dd}, J=6.9$, $14.3 \mathrm{~Hz}, 2 \mathrm{H}, \mathrm{BOM}), 4.53$ (dd, $J=12.0,15.5 \mathrm{~Hz}, 2 \mathrm{H}, \mathrm{BOM}$ ), 3.71 (ddd, $J=6.3,12.3,12.5 \mathrm{~Hz}, 1 \mathrm{H}, 21-\mathrm{H}), 2.31$ (ddd, $J=2.3,7.2,7.3 \mathrm{~Hz}, 2 \mathrm{H}, 3-\mathrm{H}$ ), 2.19 (ddd, $J=6.3,6.9,10.0 \mathrm{~Hz}, 1 \mathrm{H}, 20-\mathrm{H}$ ), 2.07 (ddd, $J=6.3,6.9,10.0 \mathrm{~Hz}$, $1 \mathrm{H}, 20-\mathrm{H}), 1.97$ (br s, $4 \mathrm{H}, 16-\mathrm{H}, 17-\mathrm{H}), 1.88$ (dd, $J=6.9,12.9 \mathrm{~Hz}, 2 \mathrm{H}$, $13-\mathrm{H}), 1.53(\mathrm{tt}, J=7.5,7.5 \mathrm{~Hz}, 2 \mathrm{H}, 2-\mathrm{H}), 1.25-1.18(\mathrm{~m}, 18 \mathrm{H}, 4-\mathrm{H}$ to $12-\mathrm{H})$, $1.09(\mathrm{~d}, J=6.3 \mathrm{~Hz}, 3 \mathrm{H}, 22-\mathrm{H}) .{ }^{13} \mathrm{C}-\mathrm{NMR}\left(125 \mathrm{MHz}, \mathrm{CDCl}_{3}\right): \delta 202.7(\mathrm{C} 1)$, 138.0 (BOM), 132.6 (C18), 130.7 (C14), 129.4 (C15), 128.3 (BOM), 127.8 (BOM), 127.5 (BOM), 126.2 (С19), 92.7 (BOM), 72.9 (С21), 69.2 (BOM), 43.8 (C2), 40.0 (C20), 32.7 (C17), 32.5 (C16), 32.5 (C13), 29.6, 29.5, 29.5, 29.4, 29.3, 29.1 (C4 to C12), 22.0 (C3), 19.8 (C22). HR-MS (ESI-TOF): $\mathrm{m} / \mathrm{z}$ calcd for $\mathrm{C}_{30} \mathrm{H}_{48} \mathrm{O}_{3} \mathrm{Na}[\mathrm{M}+\mathrm{Na}]^{+} 479.3496$, found 479.3514. IR (neat): 2924, 2854, $1727 \mathrm{~cm}^{-1}$. $[\alpha]_{\mathrm{D}}{ }^{21}-3.91\left(\right.$ c $\left.1.00, \mathrm{CHCl}_{3}\right)$.

(3S,16E,20E,23S)-Ethyl 23-benzyloxymethoxy-3-hydroxytetracosa-16,20dienthioate (9). ${ }^{1} \mathrm{H}-\mathrm{NMR}\left(500 \mathrm{MHz}, \mathrm{CDCl}_{3}\right): \delta 7.36-7.27(\mathrm{~m}, 5 \mathrm{H}, \mathrm{BOM})$, 5.51-5.34 (m, $4 \mathrm{H}, 16-\mathrm{H}, 17-\mathrm{H}, 20-\mathrm{H}, 21-\mathrm{H}), 4.80(\mathrm{dd}, J=7.5,14.3 \mathrm{~Hz}, 2 \mathrm{H}$, BOM), 4.61 (ddd, $J=11.5,15.5 \mathrm{~Hz}, 2 \mathrm{H}, \mathrm{BOM}), 4.05$ (brs, $1 \mathrm{H}, \mathrm{OH}$ ), 3.80 (ddd, $J=6.3,10.3,12.6 \mathrm{~Hz}, 1 \mathrm{H}, 3-\mathrm{H}), 2.90$ (q, $J=7.5 \mathrm{~Hz}, 2 \mathrm{H}, \mathrm{Et}), 2.74$ (dd, $J=2.9,15.5 \mathrm{~Hz}, 1 \mathrm{H}, 23-\mathrm{H}), 2.65(\mathrm{~m}, 2 \mathrm{H}, 2-\mathrm{H}), 2.28$ (ddd, $J=6.3,6.9$, $10.0 \mathrm{~Hz}, 1 \mathrm{H}, 22-\mathrm{H}$ ), 2.17 (ddd, $J=6.3,6.9,10.0 \mathrm{~Hz}, 1 \mathrm{H}, 22-\mathrm{H}$ ), 2.04 (br s, $4 \mathrm{H}$, $18-\mathrm{H}, 19-\mathrm{H}$ ), 1.95 (qn, $J=7.5 \mathrm{~Hz}, 2 \mathrm{H}, 15-\mathrm{H}), 1.55-1.25$ (m, 25H, 4-H to $14-\mathrm{H}$, Et), 1.17 (d, $J=6.3 \mathrm{~Hz}, 3 \mathrm{H}, 24-\mathrm{H}) .{ }^{13} \mathrm{C}-\mathrm{NMR}\left(125 \mathrm{MHz}, \mathrm{CDCl}_{3}\right): \delta 199.7(\mathrm{Cl})$, 138.0 (BOM), 132.7 (C20), 130.8 (C16), 129.4 (C17), 128.4 (BOM), 127.8 (BOM), 127.6 (BOM), 126.2 (С21), 92.8 (BOM), 73.0 (С23), 69.2 (BOM), 68.7 (C3), 50.6 (C2), 40.0 (C22), 36.5 (C4), 32.8 (C19), 32.6 (C18), 32.5 (C15), 29.6, 29.6, 29.6, 29.5, 29.5, 29.5, 29.5, 29.1, 25.4 (C6 to C14, Et), 23.3 (C5), 19.9 (C24), 14.6 (Et). HR-MS (ESI-TOF): $m / z$ calcd for $\mathrm{C}_{34} \mathrm{H}_{56} \mathrm{O}_{4} \mathrm{Na}[\mathrm{M}+\mathrm{Na}]^{+}$ 583.3792, found 583.3778. IR (neat): 2924, $2854,1727 \mathrm{~cm}^{-1} \cdot[\alpha]_{\mathrm{D}}^{21}+4.87$ (c $\left.1.00, \mathrm{CHCl}_{3}\right)$.

(3S,16E,20E,23S)-Ethyl 23-benzyloxymethoxy-3-hydroxytetracosa-16,20-dienoate (10). ${ }^{1} \mathrm{H}-\mathrm{NMR}\left(500 \mathrm{MHz}, \mathrm{CDCl}_{3}\right): \delta 7.35-7.27$ (m, 5H, BOM), 5.52-5.34 (m, $4 \mathrm{H}, 16-\mathrm{H}, 17-\mathrm{H}, 20-\mathrm{H}, 21-\mathrm{H}), 4.80(\mathrm{dd}, J=7.5,14.3 \mathrm{~Hz}, 2 \mathrm{H}, \mathrm{BOM}), 4.61$ (ddd, $J=12.0,15.5 \mathrm{~Hz}, 2 \mathrm{H}, \mathrm{BOM}$ ), 4.17 (q, $J=6.9 \mathrm{~Hz}, 2 \mathrm{H}, \mathrm{Et}$ ), 4.00 (brs, $1 \mathrm{H}$, $\mathrm{OH}), 3.80(\mathrm{ddd}, J=6.3,10.3,12.6 \mathrm{~Hz}, 1 \mathrm{H}, 3-\mathrm{H}), 2.94(\mathrm{~d}, J=4.0 \mathrm{~Hz}, 1 \mathrm{H}, 23-\mathrm{H})$, 2.51-2.48 (m, 2H, 2-H), 2.28 (ddd, $J=6.3,6.9,10.0 \mathrm{~Hz}, 1 \mathrm{H}, 22-\mathrm{H}$ ), 2.15 (ddd, $J=6.3,6.9,10.0 \mathrm{~Hz}, 1 \mathrm{H}, 22-\mathrm{H}$ ), 2.04 (br s, 4H, 18-H, 19-H), 1.95 (m, 2H, 15-H), 1.63-1.25 (m, 25H, 4-H to $14-\mathrm{H}, \mathrm{Et}), 1.17$ (d, $J=6.3 \mathrm{~Hz}, 3 \mathrm{H}$, 24-H). ${ }^{13} \mathrm{C}-\mathrm{NMR}\left(125 \mathrm{MHz}, \mathrm{CDCl}_{3}\right): \delta 173.1(\mathrm{C} 1), 138.0$ (BOM), 132.7 (C20), 130.8 (C16), 129.5 (C17), 128.4 (BOM), 127.8 (BOM), 127.6 (BOM), 126.2 (C21), 92.8 (BOM), 73.0 (С23), 69.3 (BOM), 68.0 (C3), $60.6(\mathrm{Et}), 41.3$ (C2), 40.0 (C22), 36.5 (C4), 32.8 (C19), 32.6 (C18), 32.6 (C15), 29.6, 29.6, 29.6, 29.5, 29.2 (C6 to C14), 25.5 (C5), 19.9 (C24), 14.2 (Et). HR-MS (ESI-TOF): $\mathrm{m} / \mathrm{z}$ calcd for $\mathrm{C}_{34} \mathrm{H}_{56} \mathrm{O}_{5} \mathrm{Na}[\mathrm{M}+\mathrm{Na}]^{+} 567.4020$, found 567.4000. IR (neat): 3464, $2924,2854,1728 \mathrm{~cm}^{-1} \cdot[\alpha]_{\mathrm{D}}{ }^{20}+4.27\left(c 1.02, \mathrm{CHCl}_{3}\right)$.

(3S,16E,20E,23S)-Ethyl 23-hydroxy-3-(p-methoxybenzyloxy)tetracosa-16, 20-dienoate (12). ${ }^{1} \mathrm{H}-\mathrm{NMR}\left(500 \mathrm{MHz}, \mathrm{CDCl}_{3}\right): \delta 7.24$ (m, 2H, PMB), 6.86 (m, 2H, PMB), 5.58-5.34 (m, 4H, 16-H, 17-H, 20-H, 21-H), 4.46 (dd, $J=10.9$, $15.8 \mathrm{~Hz}, 2 \mathrm{H}, \mathrm{PMB}$ ), 4.14 (dq, $J=1.7,7.5 \mathrm{~Hz}, 2 \mathrm{H}, \mathrm{Et}$ ), 3.88-3.76 (m, 5H, 3-H, 23-H, PMB), 2.58 (q, $J=7.5 \mathrm{~Hz}, 1 \mathrm{H}, 22-\mathrm{H}), 2.44(\mathrm{dd}, J=5.7,14.5 \mathrm{~Hz}, 1 \mathrm{H}$, 22-H), 2.23-2.17 (m, 2H, 2-H), 2.13-2.04 (m, 4H, 18-H, 19-H), 1.96 (q, $J=6.9 \mathrm{~Hz}, 2 \mathrm{H}, 15-\mathrm{H}), 1.63-1.49(\mathrm{~m}, 2 \mathrm{H}, 4-\mathrm{H}), 1.43-1.24(\mathrm{~m}, 23 \mathrm{H}, 5-\mathrm{H}$ to $14-\mathrm{H}, \mathrm{Et}), 1.18$ (d, J=6.3 Hz, 3H, 24-H). ${ }^{13} \mathrm{C}-\mathrm{NMR}\left(125 \mathrm{MHz}, \mathrm{CDCl}_{3}\right)$ : $\delta 171.9$ (C1), 159.1 (PMB), 134.2 (C20), 131.1 (C16), 130.7 (C17), 129.4 (PMB), 129.3 (PMB), 126.2 (C21), 113.7 (PMB), 75.8 (PMB), 71.2 (C23), 67.0 (C3), 60.4 (Et), 55.3 (PMB), 42.5 (C2), 40.0 (C22), 34.5 (C4), 32.7 (C19), 32.6 (C18), 32.5 (C15), 29.7, 29.7, 29.6, 29.6, 29.6, 29.5, 29.2 (C6 to C14), 25.2 (C5), 22.6 (C24), 14.2 (Et). HR-MS (ESI-TOF): $m / z$ calcd for $\mathrm{C}_{34} \mathrm{H}_{56} \mathrm{O}_{5} \mathrm{Na}[\mathrm{M}+\mathrm{Na}]^{+}$ 567.4020 , found 567.4010. IR (neat): $3464,2924,2854,1728 \mathrm{~cm}^{-1} \cdot[\alpha]_{\mathrm{D}}{ }^{20}$ $+2.65\left(\right.$ c $\left.1.00, \mathrm{CHCl}_{3}\right)$.

(3S, 16E,20E,23S)-23-Hydroxy-3-(p-methoxybenzyloxy)tetracosa-16,20-dienoic acid (13). ${ }^{1} \mathrm{H}-\mathrm{NMR}\left(500 \mathrm{MHz}, \mathrm{CDCl}_{3}\right): \delta 7.25(\mathrm{~m}, 2 \mathrm{H}, \mathrm{PMB}), 6.87(\mathrm{~m}, 2 \mathrm{H}$, PMB), 5.56-5.34 (m, 4H, 16- H, 17-H, 20-H, 21-H), 4.51 (dd, $J=10.9,14.3 \mathrm{~Hz}$, 2H, PMB), 3.86-3.76 (m, 5H, 3-H, 23-H, PMB), 2.58 (dq, J=5.2, $14.9 \mathrm{~Hz}, 2 \mathrm{H}$, $22-\mathrm{H}), 2.19(\mathrm{dt}, J=5.2,16.3 \mathrm{~Hz}, 2 \mathrm{H}, 2-\mathrm{H}), 2.19-2.05(\mathrm{~m}, 4 \mathrm{H}, 18-\mathrm{H}, 19-\mathrm{H})$, $1.96(\mathrm{dd}, J=6.3,13.8 \mathrm{~Hz}, 2 \mathrm{H}, 15-\mathrm{H}), 1.63(\mathrm{~m}, 1 \mathrm{H}, 4-\mathrm{H}), 1.55(\mathrm{~m}, 1 \mathrm{H}, 4-\mathrm{H})$, $1.35-1.25$ (m, 20H, 5-H to $14-\mathrm{H}), 1.18(\mathrm{~d}, J=6.3 \mathrm{~Hz}, 3 \mathrm{H}, 24-\mathrm{H}) .{ }^{13} \mathrm{C}-\mathrm{NMR}$ (125 MHz, $\mathrm{CDCl}_{3}$ ): $\delta 174.0(\mathrm{C} 1), 159.4$ (PMB), 134.3 (C20), 131.1 (C16), 129.8 (C17), 129.6 (PMB), 129.4 (PMB), 126.1 (C21), 113.9 (PMB), 75.3 (PMB), 71.2 (C23), 67.1 (C3), 55.3 (PMB), 42.5 (C2), 39.0 (C22), 33.9 (C4), 32.7 (C19), 32.6 (C18), 32.5 (C15), 29.7, 29.6, 29.6, 29.5, 29.5, 29.1 (C6 to C14), 25.0 (C5), 22.5 (C24). HR-MS (ESI-TOF): $\mathrm{m} / z$ calcd for $\mathrm{C}_{32} \mathrm{H}_{52} \mathrm{O}_{5} \mathrm{Na}[\mathrm{M}+\mathrm{Na}]^{+}$ 539.3707, found 539.3727. IR (neat): $3464,2924,2854,1728 \mathrm{~cm}^{-1} \cdot[\alpha]_{\mathrm{D}}{ }^{20}$ $+15.1\left(\right.$ ( $\left.1.00, \mathrm{CHCl}_{3}\right)$.

(3S, 16E,20E,23S)-3-(p-Methoxybenzyloxy)-23-methyltricosa-16,20-dienolide (14). ${ }^{1} \mathrm{H}-\mathrm{NMR}\left(500 \mathrm{MHz}, \mathrm{CDCl}_{3}\right): \delta 7.26$ (m, 2H, PMB), 6.87 (m, 2H, PMB), 5.475.29 (m, 4H, 16-H, 17-H, 20-H, 21-H), 4.91 (dt, $J=6.0,6.5 \mathrm{~Hz}, 1 \mathrm{H}, 3-\mathrm{H}), 4.47$ (dd, $J=11.5,40.4 \mathrm{~Hz}, 2 \mathrm{H}, \mathrm{PMB}), 3.83-3.77$ (m, 4H, 23-H, PMB), 2.65 (dd, $J=5.4,14.0 \mathrm{~Hz}, 1 \mathrm{H}, 2-\mathrm{H}$ ), 2.43 (dd, $J=7.7,14.0 \mathrm{~Hz}, 1 \mathrm{H}, 2-\mathrm{H}$ ), 2.24 (ddd, $J=5.7,6.3,7.5 \mathrm{~Hz}, 2 \mathrm{H}, 22-\mathrm{H}$ ), 2.04 (br s, $4 \mathrm{H}, 18-\mathrm{H}, 19-\mathrm{H}$ ), 1.99 (dd, $J=6.3$, $11.7 \mathrm{~Hz}, 2 \mathrm{H}, 15-\mathrm{H}), 1.39-1.27(\mathrm{~m}, 22 \mathrm{H}, 4-\mathrm{H}$ to $14-\mathrm{H}), 1.19(\mathrm{~d}, J=6.0 \mathrm{~Hz}$, $3 \mathrm{H}, 24-\mathrm{H}) .{ }^{13} \mathrm{C}-\mathrm{NMR}\left(125 \mathrm{MHz}, \mathrm{CDCl}_{3}\right): \delta 170.9$ (C1), 159.1 (PMB), 133.5 (C20), 130.8 (C16), 130.6 (C17), 129.8 (PMB), 129.3 (PMB), 125.2 (C21), 113.7 (PMB), 75.5 (PMB), 70.7 (C23), 70.6 (C3), 55.3 (PMB), 40.0 (C2), 39.0 (C22), 33.9 (C4), 32.7 (C19), 32.4 (C18), 31.8 (C15), 28.8, 28.6, 28.5, 28.4, 28.2, 28.2, 27.5 (C6 to C14), 24.4 (C5), 19.4 (C24). HR-MS (ESI-TOF): $\mathrm{m} / \mathrm{z}$ calcd for $\mathrm{C}_{32} \mathrm{H}_{50} \mathrm{O}_{4} \mathrm{Na}[\mathrm{M}+\mathrm{Na}]^{+} 521.3601$, found 521.3600. IR (neat): 2924, $2854,1728 \mathrm{~cm}^{-1} \cdot[\alpha]_{\mathrm{D}}^{20}-4.63\left(\right.$ c $\left.1.00, \mathrm{CHCl}_{3}\right)$.

(3S,16E,20E,23S)-3-Hydroxy-23-methyltricosa-16,20-dienolide (15). ${ }^{1} \mathrm{H}-\mathrm{NMR}$ $\left(500 \mathrm{MHz}, \mathrm{CDCl}_{3}\right): \delta 5.51-5.34(\mathrm{~m}, 4 \mathrm{H}, 16-\mathrm{H}, 17-\mathrm{H}, 20-\mathrm{H}, 21-\mathrm{H}), 5.00$ (dt, $J=1.5,6.0 \mathrm{~Hz}, 1 \mathrm{H}, 3-\mathrm{H}), 3.94(\mathrm{~m}, 1 \mathrm{H}, 23-\mathrm{H}), 2.54-2.39(\mathrm{~m}, 2 \mathrm{H}, 2-\mathrm{H})$, 2.31-2.17 (m, 2H, 22-H), 2.05 (br s, $4 \mathrm{H}, 18-\mathrm{H}, 19-\mathrm{H}), 2.00(\mathrm{~d}, J=5.5 \mathrm{~Hz}, 2 \mathrm{H}$, $15-\mathrm{H}), 1.58-1.27(\mathrm{~m}, 22 \mathrm{H}, 4-\mathrm{H}$ to $14-\mathrm{H}), 1.24(\mathrm{~d}, J=6.0 \mathrm{~Hz}, 3 \mathrm{H}, 24-\mathrm{H})$. ${ }^{13} \mathrm{C}-\mathrm{NMR}\left(125 \mathrm{MHz}, \mathrm{CDCl}_{3}\right): \delta 172.2(\mathrm{C} 1), 133.7(\mathrm{C} 20), 130.8(\mathrm{C} 16), 129.8$ (C17), 125.0 (C21), 70.8 (C23), 68.2 (C3), 41.4 (C2), 39.0 (C22), 36.1 (C4), 32.9 (C19), 32.5 (C18), 31.9 (C15), 28.7, 28.6, 28.4, 28.2, 28.2, 28.2, 28.1, 28.0, 27.5 (C6 to C14), 24.7 (C5), 19.6 (C24). HR-MS (ESI-TOF): $\mathrm{m} / \mathrm{z}$ calcd for 
$\mathrm{C}_{24} \mathrm{H}_{42} \mathrm{O}_{3} \mathrm{Na}[\mathrm{M}+\mathrm{Na}]^{+}$401.3026, found 401.3008. IR (neat): 3410, 2924, 2854, $1728 \mathrm{~cm}^{-1} \cdot[\alpha]_{\mathrm{D}}^{22}-13.5\left(c 0.87, \mathrm{CHCl}_{3}\right)$.

(3S, 16E,20E,23S)-Eushearilide (2). ${ }^{1} \mathrm{H}-\mathrm{NMR} \quad\left(500 \mathrm{MHz}, \quad \mathrm{CD}_{3} \mathrm{OD}\right): \quad \delta 5.50$ $(\mathrm{m}, 2 \mathrm{H}, 20-\mathrm{H}), 5.39(\mathrm{~m}, 2 \mathrm{H}, 21-\mathrm{H}), 5.37$ (br s, 4H, 16- H, 17-H), 4.88 (tq, $J=6.8,6.5 \mathrm{~Hz}, 1 \mathrm{H}, 23-\mathrm{H}), 4.54(\mathrm{~m}, 1 \mathrm{H}, 3-\mathrm{H}), 4.26\left(\mathrm{~m}, 2 \mathrm{H}, 1^{\prime}-\mathrm{H}\right), 3.63$ (m, 2H, 2'-H), $3.22\left(\mathrm{~s}, 9 \mathrm{H}, \mathrm{NMe}_{3}\right), 2.82(\mathrm{dd}, J=4.3,14.5 \mathrm{~Hz}, 1 \mathrm{H}, 2-\mathrm{H}), 2.54$ (dd, $J=8.714 .5 \mathrm{~Hz}, 1 \mathrm{H}, 2-\mathrm{H}$ ), 2.28 (ddd, $J=6.8,6.8,13.5 \mathrm{~Hz}, 1 \mathrm{H}, 22-\mathrm{H}$ ), 2.22 (ddd, $J=6.8,6.8,13.5 \mathrm{~Hz}, 1 \mathrm{H}, 22-\mathrm{H}$ ), 2.07 (br s, $4 \mathrm{H}, 18-\mathrm{H}, 19-\mathrm{H}$ ), 2.00 (br dd, $J=5.8,9.0 \mathrm{~Hz}, 2 \mathrm{H}, 15-\mathrm{H}), 1.65$ (ddt, $J=14.5,14.5,7.5 \mathrm{~Hz}, 2 \mathrm{H}, 4-\mathrm{H}), 1.50-1.40$ $(\mathrm{m}, 2 \mathrm{H}, 5-\mathrm{H}), 1.36(\mathrm{~m}, 2 \mathrm{H}, 14-\mathrm{H}), 1.30(\mathrm{br} \mathrm{s}, 16 \mathrm{H}, 6-\mathrm{H}$ to $13-\mathrm{H}), 1.19$ (d, $J=6.5 \mathrm{~Hz}, 3 \mathrm{H}, 23-\mathrm{Me}) .{ }^{13} \mathrm{C}-\mathrm{NMR}\left(125 \mathrm{MHz}, \mathrm{CD}_{3} \mathrm{OD}\right): \delta 171.8(\mathrm{Cl}), 134.7$ (C20), 131.8 (C16), 131.2 (C17), 126.5 (C21), 74.1 (C3), 72.3 (C23), 67.5 $\left(\mathrm{C}^{\prime}\right), 60.3\left(\mathrm{Cl}^{\prime}\right), 54.7\left(\mathrm{C} 27\left(\mathrm{NMe}_{3}\right)\right), 41.9(\mathrm{C} 2), 40.0(\mathrm{C} 22), 36.1(\mathrm{C} 4), 33.9$ (C19), 33.6 (C18), 32.8 (C15), 30.1 (C6), 29.5 (C14), 29.8, 29.7, 29.6, 29.5, 29.4, 29.2 (C7 to C12), 28.5 (C13), 25.4 (C5), 19.7 (C24). HR-MS (ESI-TOF): $m / z$ calcd for $\mathrm{C}_{29} \mathrm{H}_{54} \mathrm{NO}_{6} \mathrm{PNa}[\mathrm{M}+\mathrm{Na}]^{+} 566.3586$, found 566.3592 . IR (neat): 3432 , 2954, 2537, 2090, $1728 \mathrm{~cm}^{-1} \cdot[\alpha]_{\mathrm{D}}{ }^{25}+11.3\left(c 0.85, \mathrm{CH}_{3} \mathrm{OH}\right)$.

\section{Synthesis of 5 by using Schlosser-modified Wittig reaction}

To a cooled $\left(-78^{\circ} \mathrm{C}\right)$ solution of $4(16 \mathrm{mg}, 0.048 \mathrm{mmol})$ in tetrahydrofuran $(1.0 \mathrm{ml})$ were added $\mathrm{LiBr}(45 \mathrm{mg}, 0.052 \mathrm{mmol})$ and $\mathrm{PhLi}(1.6 \mathrm{M}$ in butyl ether, $0.03 \mathrm{ml}, 0.048 \mathrm{mmol})$. After the solution was stirred at that temperature for $30 \mathrm{~min}$, then the solution was added dropwise via cold cannula to a solution of $3(30 \mathrm{mg}, 0.048 \mathrm{mmol})$ in diethyl ether $(1.0 \mathrm{ml})$ at $-78^{\circ} \mathrm{C}$. After completion of the dripping, $\mathrm{PhLi}(1.6 \mathrm{M}$ in butyl ether, $0.03 \mathrm{ml}, 0.048 \mathrm{mmol}$ ) was added to the mixture, and the solution was stirred for $3 \mathrm{~h}$. After successive addition of $\mathrm{HCl}$ $\left(1.0 \mathrm{M}\right.$ in $\left.\mathrm{Et}_{2} \mathrm{O}, 0.08 \mathrm{ml}, 0.080 \mathrm{mmol}\right)$ and $\mathrm{KO}^{t} \mathrm{Bu}\left(1.0 \mathrm{M}\right.$ in $\mathrm{Et}_{2} \mathrm{O}, 0.06 \mathrm{ml}$, $0.057 \mathrm{mmol}$ ), the reaction mixture was stirred for $1 \mathrm{~h}$ at $-78^{\circ} \mathrm{C}$. The reaction mixture was quenched with water, and the organic layer was separated and the aqueous layer was extracted with diethyl ether three times. The combined organic layer was washed with water and brine and dried over sodium sulfate. After evaporation of the solvent, the crude product was purified by TLC on silica gel (hexane/ethyl acetate $=10 / 1)$ to afford $5(21 \mathrm{mg}, 76 \%)$.

\section{Synthesis of 14 by using MNBA-mediated macrolactonization with DMAP}

To a solution of MNBA ( $20 \mathrm{mg}, 57.9 \mu \mathrm{mol})$ and DMAP ( $33 \mathrm{mg}, 0.27 \mathrm{mmol})$ in dichloromethane $(18.0 \mathrm{ml})$ at room temperature was slowly added a solution of the seco acid $13(23 \mathrm{mg}, 44.5 \mu \mathrm{mol})$ in dichloromethane $(4.0 \mathrm{ml})$ with a mechanically driven syringe over a $12 \mathrm{~h}$ period. After cooling to $0{ }^{\circ} \mathrm{C}$, saturated aqueous sodium hydrogen carbonate was added. The organic layer was separated, the aqueous layer was extracted with dichloromethane, and the organic layer was washed with brine and water and dried over sodium sulfate. The combined organic layer was washed with water and brine and dried over sodium sulfate. After evaporation of the solvent, the crude product was purified by TLC on silica gel (hexane/ethyl acetate $=6 / 1)$ to afford $14(15 \mathrm{mg}, 67 \%)$.

\section{Antimicrobial assay}

The antimicrobial assay against S. aureus (NBRC 12732), A. niger (NBRC 105649) and T. mentagrophytes (NBRC 5466) was performed using the disk diffusion method according to the Clinical and Laboratory Standards Institute. ${ }^{13}$ Details of the assay method are described in Supplementary Information.

\section{CONFLICT OF INTEREST}

The authors declare no conflict of interest.

\section{ACKNOWLEDGEMENTS}

This study was partly supported by a Research Grant from the Center for Chirality and Grants-in-Aid for Scientific Research from the Ministry of Education, Science, Sports and Culture, Japan.
1 Hosoe, T. et al. A new antifungal macrolide, eushearilide, isolated from Eupenicillium shearii. J. Antibiot. 59, 597-600 (2006).

2 Shiina, I. \& Fujimori, K. Synthetic studies on eushearilide. Abstracts of Papers, 89th Annual Meeting of the Chemical Society of Japan, 27-30 March 2009, Funabashi, Chiba, Japan; Vol. 2, 2J431.

3 Shiina, I. \& Fujimori, K. Construction of the 24-membered lactone moiety of eushearilide. Abstracts of Papers, 90th Annual Meeting of the Chemical Society of Japan, 26-29 March 2010, Higashiosaka, Osaka, Japan; Vol. 2, 2A530.

4 Tonoi, T. \& Shiina, I. Asymmetric total synthesis of (3R,23R)-eushearilide. Abstracts of Papers, 92th Annual Meeting of the Chemical Society of Japan, 25-28 March 2012, Yokohama, Kanagawa, Japan; Vol. 2, 2 E104.

5 Tonoi, T., Kawahara, R., Tanaka, A. \& Shiina, I. Total synthesis of $(16 Z, 20$ E)eushearilide. Abstracts of Papers, 94th Annual Meeting of the Chemical Society of Japan, 27-30 March 2014, Nagoya, Aichi, Japan; Vol. 2, 3H709.

6 Yamauchi, T. et al. Total synthesis of the proposed structures of eushearilide. Heterocycles 88, 1175-1189 (2014).

7 Shiina, I., Ibuka, R. \& Kubota, M. A new condensation reaction for the synthesis of carboxylic esters from nearly equimolar amounts of carboxylic acids and alcohols using 2-methyl-6-nitrobenzoic anhydride. Chem. Lett. 31, 286-287 (2002).

8 Shiina, I., Kubota, M. \& Ibuka, R. A novel and efficient macrolactonization of $\omega$-hydroxycarboxylic acids using 2-methyl-6-nitrobenzoic anhydride (MNBA). Tetrahedron Lett. 43, 7535-7539 (2002).

9 Shiina, I., Kubota, M., Oshiumi, H. \& Hashizume, M. An effective use of benzoic anhydride and its derivatives for the synthesis of carboxylic esters and lactones: a powerful and convenient mixed anhydride method promoted by basic catalysts. J. Org. Chem. 69, 1822-1830 (2004).

10 Shiina, I. \& Hashizume, M. Synthesis of (9E)-isoambrettolide, a macrocyclic musk compound, using the effective lactonization promoted by symmetric benzoic anhydrides with basic catalysts. Tetrahedron 62, 7934-7939 (2006).

11 Shiina, I., Kikuchi, T. \& Sasaki, A. The first total synthesis of (-) and (+)-2-hydroxy-24oxooctacosanolide using an effective lactonization. Org. Lett. 8, 4955-4958 (2006).

12 Shiina, I. Total synthesis of natural 8- and 9-membered lactones: recent advancements in medium-sized ring formation. Chem. Rev. 107, 239-273 (2007).

13 Shiina, I., Sasaki, A., Kikuchi, T. \& Fukui, H. Total synthesis of 2-hydroxytetracosanolide and 2-hydroxy-24-oxooctacosanolide by using an effective lactonization. Chem. Asian J. 3, 462-472 (2008).

14 Shiina, I. An adventurous synthetic journey with MNBA from its reaction chemistry to the total synthesis of natural products. Bull. Chem. Soc. Jpn. 87, 196-233 (2014).

15 Tonoi, T. et al. Total synthesis of $(3 R, 16 E, 20 E, 23 R)-(-)$-eushearilide and structural determination of naturally occurring eushearilide. Tetrahedron Lett. 56, 1356-1359 (2015).

16 Schlosser, M. \& Christmann, K. F. Olefinierungen mit phosphor-yliden. I. Mechanismus und stereochemie der Wittig-reaktion . Liebigs Ann. Chem. 708, 1-35 (1967).

17 Schlosser, M., Ba, T. H. \& Schaub, B. The betaine-ylid route to trans-alkenols. Tetrahedron Lett. 26, 311-314 (1985).

18 Wang, Q., Deredas, D., Huynh, C. \& Schlosser, M. Sequestered alkyllithiums: why phenyllithium alone is suitable for betaine-ylide generation. Chemistry $\mathbf{9}$, 570-574 (2003).

19 de Haan, J. W. \& van de Ven, L. J. M. Configurations and conformations in acyclic, unsaturated hydrocarbons. A $13 C$ NMR study. Org. Magn. Reson. 5, 147-153 (1973).

20 Lee, Y. et al. Cyclic bis-1,3-dialkylpyridiniums from the sponge Haliclona sp. Mar. Drugs 10, 2126-2137 (2012).

21 Damodaran, V., Ryan, J. L. \& Keyzers, R. A. Cyclic 3-alkyl pyridinium alkaloid monomers from a New Zealand Haliclona sp. marine sponge. J. Nat. Prod. 76, 1997-2001 (2013).

22 Czajkowska-Szczykowska, D., Jastrzebska, I., Santillan, R. \& Morzycki, J. W. The synthesis of disteroidal macrocyclic molecular rotors by an RCM approach. Tetrahedron 70, 9427-9435 (2014).

23 Mukaiyama, T., Kobayashi, S., Uchiro, H. \& Shiina, I. Catalytic asymmetric aldol reaction of silyl enol ethers with aldehydes by the use of chiral diamine coordinated tin (II) triflate. Chem. Lett. 19, 129-132 (1990).

24 Kobayashi, S., Uchiro, H., Fujishita, Y., Shiina, I. \& Mukaiyama, T. Asymmetric aldol reaction between achiral silyl enol ethers and achiral aldehydes by use of a chiral promoter system. J. Am. Chem. Soc. 113, 4247-4252 (1991).

25 Shiina, I. Asymmetric Mukaiyama aldol reactions using chiral diamine-coordinated Sn (II) triflate: development and application to natural product synthesis. Chem. Rec. 14, 144-183 (2014).

26 Qin, D., Byun, H.-S. \& Bittman, R. Synthesis of plasmalogen via 2,3-bis-0-(4'methoxybenzyl)-sn-glycerol. J. Am. Chem. Soc. 121, 662-668 (1999).

27 National Committee for Clinical Laboratory Standards. Standard Methods for Dilution Antimicrobial Susceptibility Tests for Bacteria that Grow Aerobically, Approved Standards (NCCLS, Villanova, PA, USA, 1983).

28 National Committee for Clinical Laboratory Standards. Methods for Dilution Antimicrobial Susceptibility Tests for Bacteria that Grow Aerobically, Second Edition, Tentative Standard (NCCLS, Villanova, PA, USA, 1988).

29 Takaoka, Y. Examination about the combined use effect of trichlosan and urea to fungus. Jpn. J. Chemother. 63, 24-26 (2003). 